\title{
Controlled adsorption and release onto calcium phosphates materials and drug delivery applications
}

\author{
A. Barroug ${ }^{1}$, H. Noukrati ${ }^{1,2}$, F. Errassifi' ${ }^{3}$ S. Sarda ${ }^{3}$, S. Cazalbou ${ }^{3}$, C. Combes ${ }^{2}$, C. Rey ${ }^{2}$ \\ and M.J. Glimcher ${ }^{4}$ \\ ${ }^{1}$ LPCME-CNRST-URAC 20, FSSM, BP. 2390, Université Cadi Ayyad, 40000 Marrakech, Maroc \\ 2 Université de Toulouse, CIRIMAT, UPS-INPT-CNRS, ENSIACET, 4 allée Emile Monso, BP. 44362, 31030 \\ Toulouse, France \\ 3 Université de Toulouse, CIRIMAT, UPS-INPT-CNRS, Université Paul Sabatier, Faculté de Pharmacie, \\ 118 route de Narbonne, 31062 Toulouse Cedex 9, France \\ ${ }^{4}$ Laboratory for the Study of Skeletal Disorders and Rehabilitation, Department of Orthopaedic Surgery, Harvard \\ Medical School, Children's Hospital, 300 Longwood Avenue, Boston, MA 02115, USA
}

\begin{abstract}
The adsorptive properties of synthetic calcium phosphates analogous to bone mineral were examined with respect to cisplatin and risedronate, two biological active drugs; the uptake and release experiments were carried out under various conditions in order to understand the basic mechanism of interaction. The effect of temperature and solution composition were highlighted and discussed. The adsorption results obtained for the therapeutic agents demonstrated that, depending on the conditions investigated (nature of the sorbent, concentration range, ionic composition, temperature...), the shape of the isotherms is of Freundlich or Langmuir type. The adsorption is described as an ion-exchange process in dilute solutions, while the interaction appears to be reactive for concentrated solutions (dissolution of mineral ions from the apatite substrate and formation of soluble calcium complex and/or precipitation of calcium salts involving sorbate molecules). The information gained on the surface reactivity of calcium phosphate were exploited to associate an antibiotic to calcium phosphate cements for drug delivery applications. The specimens were obtained by combination of calcium phosphate and calcium carbonate powders upon mixing with water. The physicochemical properties of the paste were altered by the drug loading method (in the liquid or solid phase). Thus, a dose-dependent effect was noticed for the paste setting time, hardening and the release process.
\end{abstract}

\section{INTRODUCTION}

In the last decades, calcium phosphate $(\mathrm{CaP})$ materials have received major attention in the biomedical field mainly for their compositional resemblance with the mineral phase of bone and their biological properties. Thus, considerable effort and large number of studies have been devoted to develop CaP based bioceramics in vivo for bone replacement or repair as well as the use of these materials as carriers to deliver therapeutic agents in the skeletal systems [1-3]. However, the phenomena (interaction mechanisms and driving forces) occurring at the interface between synthetic $\mathrm{CaP}$ and the surrounding environments remain not totally controlled. The objective of this work is (i) to determine the in vitro basic binding and release profiles and mechanisms of interaction of pharmaceuticals with biomimetic calcium phosphates nanoparticles, and (ii) to report in a preliminary study on the preparation of a cement and its combination with antibiotics for the treatment of bone infections.

\section{MATERIALS AND METHODS}

Several apatitic calcium phosphates with different physicochemical characteristics were used in this study. The specimens were prepared under various synthesis conditions (temperature, $\mathrm{pH}$, degree of saturation ...), by double decomposition according to methods described previously $[4,5]$. The precipitates were removed by filtration, washed with de-ionized water and lyophilized overnight. The powders were characterized by means of $\mathrm{X}$-ray diffraction, Fourier transform infrared spectroscopy, and chemical analyses. The specific surface area of the samples were measured by nitrogen adsorption according to the BET method.

The adsorption experiments were performed with two therapeutics agents: (i) risedronate sodium salt, a bisphosphonate used in the prevention and treatment of several diseases related to bone tissue such as osteoporosis [6] and (ii) cisplatin (cis-diamminedichloroplatinum CDDP) one the most active agent in the treatment of bone cancer, osteosarcoma [7].

The uptake and release tests of the biologically active drugs were carried out in different conditions in order to determine the effect of temperature and solution composition. For this, the drug was dispersed in the desired medium (solution containing potassium chloride and/or mineral ions, tris buffer, phosphate buffer (PB), phosphate buffer saline (PBS)) and was added to the apatite powder. After an equilibration period, the solids (XRD, NMR, FTIR and Raman spectroscopies) and the solutions (UV spectroscopy, ICP-AES) were analyzed and the amount of drugs loaded was determined from the equilibrium concentration in solution as described in the literature $[4,5]$.

The preparation of the cement was performed by mixing two metastable phases of calcium phosphate and 


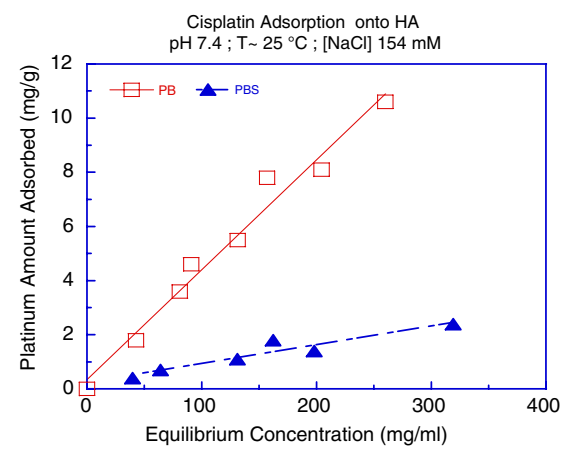

Figure 1. Adsorption isotherms of cisplatin onto hydroxyapatite (HA) in PB and PBS at $25^{\circ} \mathrm{C}$.

calcium carbonate. The starting reactants were synthesized and characterized by means of different techniques in order to control the formulation of the cement. The antibiotic, fusidic acid (FA) or its derivative, the sodium fusidate (SF), was associated with the prepared cement $(C)$. For this, the drug was incorporated either in the solid or liquid phase of the cement, leading to final products referred to as FA-C and $\mathrm{NaF}-\mathrm{C}$, respectively.

\section{RESULTS AND DISCUSSION}

Typical X-ray diffraction patterns (XRD) and infrared spectra characteristics of apatite powders were obtained for the prepared specimens. Chemical analyses showed that the solid phases were calcium-deficient apatites.

The adsorption of risedronate molecules on apatite surface as a function of contact time showed that the process occurred very rapidly (less than one hour) in $\mathrm{KCl}$ solution $(1 \mathrm{mM})$, while it was relatively slow when phosphate species were added to the medium. Besides, the adsorption kinetic of cisplatin by apatite crystals revealed that the uptake became constant after a few minutes of incubation in tris buffer, a period of 3 hours in the phosphate buffer and only after $30 \mathrm{~h}$ in the last medium containing $9 \%$ of $\mathrm{NaCl}$ (PBS).

The adsorption tests performed with cisplatin at $25^{\circ} \mathrm{C}$ revealed (Fig. 1) that only 5-6\% of the initially available drug was bound to the apatite crystals equilibrated in phosphate buffer saline, while there was significantly more binding in phosphate buffer solutions (22-25\%). Additionally, the amount loaded in PB increased linearly with increasing concentrations of cisplatin in the equilibrating solutions whereas, the amount of cisplatin loaded at $37^{\circ} \mathrm{C}$ approached a plateau of saturation. On the other hand, the evolution of the amount of risedronate adsorbed as a function of its equilibrium concentration in solution, at $25^{\circ} \mathrm{C}$ and $37^{\circ} \mathrm{C}$, showed a distinct initial sharp increase related to the affinity of the drug for the support and an adsorption plateau corresponding to the saturation of the adsorption sites. The highest adsorption capacity for the therapeutic agents was observed at physiological temperature, compared with room temperature.

Examination of the adsorption isotherms suggested that cisplatin uptake at $25^{\circ} \mathrm{C}$ can be described by a Freundlich type isotherm like that of simple molecules weakly interacting with apatite surface such as serine [8] and glycine or acetic acid [9]. Conversely, the evolution of the risedronate adsorption data which can be expressed as a Langmuir isotherm, showed that the plateau was reached at relatively low concentrations, pointing out to a high affinity between risedronate molecules and apatites surface.

For the temperature-dependence of the adsorption, the uptake of large amounts of drugs as a function of temperature implied an increase of the adsorbing sites, most likely caused by the dehydration of the drugs and calcium-phosphate surface during adsorption. Thus, Rill and al. [10] determined the thermodynamic parameters of hydroxyaptite interaction with some complexes containing functional molecules and showed that the process is endothermic and entropy-driven, due to the release of water molecules from the solubilized complexes as well as from the polar apatite surface upon adsorption. A similar dependence on temperature was previously reported for the uptake of phosphoproteins by hydroxyapatite [11].

The adsorption of the active drugs was not reversible by simple dilution of the solution and the corresponding decrease in the equilibrium concentration of the adsorbate. However, the experiments showed that the presence of chloride ions and phosphate species, which inhibited respectively the uptake of cisplatin and risedronate, were essential for their release. Conversely, the evolution of the mineral ions content of the solution after contact of the specimens with the drugs indicated that the risedronate uptake is associated with the release of phosphate ions. Based on these observations, the adsorption in dilute solutions can be described as an ion exchange reaction in which the risedronate ions from the solution substitute for the phosphate ions from the apatitic surface, which share the same ionic sites at the crystals surface. Similar trends were noted for the interaction of apatite surface with anionic molecules such as catalase, succinylated lyzozyme, and phosphoserine [12]. Furthermore, the experiments performed in high concentrations range (data not shown hear) indicated that the process is reactive, manifested by the dramatic increase of mineral ions in solution: the interaction of sorbate molecules with apatite surface caused the dissolution of mineral ions and the subsequent formation of soluble calcium complex and/or precipitation of calcium salts. However, no formation of a separate phase was detected under the investigated conditions by XRD suggesting the possible formation of an amorphous calcium salt.

The in vitro optimisation and control of the uptake and release driving forces put forward the great potential of calcium phosphate materials as carriers to deliver therapeutic agents in the skeletal systems. In this contest, this preliminary study reported on the formulation of mixed cements, obtained by the combination of calcium phosphate and calcium carbonate powders upon mixing with water, in addition to the antibiotic incorporation to the cements under different forms and concentrations. In all cases, the end products of the cement setting reaction were mainly composed of nanocrystalline carbonated apatite analogous to bone mineral, with some residual calcium carbonate reacting phase (Fig. 2). The physicochemical 


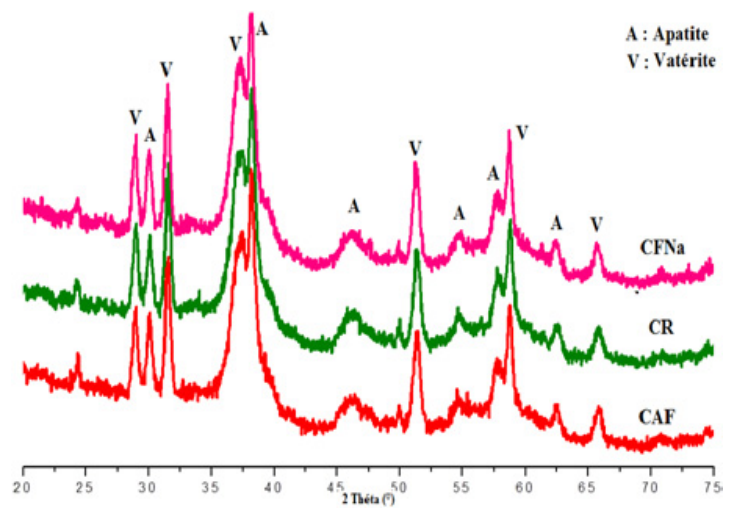

Figure 2. $\mathrm{X}$ ray diffraction patterns of the reference $(\mathrm{C})$ and drugloaded (FA-C and NaF-C) cement. The phase $\mathrm{V}$ referred to the remaining reactant (vaterite).

properties of the prepared cement were affected by the way of drug incorporation. To this regards, the setting reaction of the cements was influenced by the presence of the drug in the liquid or in the powder phase, affecting the final features of the material. Furthermore, a dosedependent effect was noticed for the paste setting time and hardening. The in vitro release tests conducted in Tris buffer showed satisfactory drug liberation profiles under the conditions examined, with a positive effect of the dose; thus, the cement containing $6 \%$ of the drug released approximately $70 \%$ of the drug (per weight) after 18 days of release test at $37^{\circ} \mathrm{C}$.

In conclusion, this study reported on the interaction under various conditions of apatitic calcium phosphate with biological active drugs with the aim of applications in the biomedical field. To this regards, antibiotics were associated to $\mathrm{CaP}$ based cements. The preliminary results obtained appear to be very promising and work is in progress to evaluate the dose effect on the physicochemical properties of the paste and the subsequent correlation with the release kinetics.

A part of this work was supported by the "Volubilis program MA/11/263".

\section{References}

[1] M.P. Ginebra, C. Canal, M. Espanol, D. Pastorino, and E.B. Montufar, Adv. Drug Deliv. Rev. 64, 1090 (2012).

[2] C. Rey et al., Bioactive Ceramics: Physical Chemistry, in Comprehensive Biomaterials, volume 1, 187-221 (Elsevier Ltd, 2011).

[3] S.J. Kalita, A. Bhardwaj, and H.A. Bhatt: Mater. Sci. Eng. C27, 449 (2007).

[4] F. Errassifi, Doctoral Thesis, Cadi Ayyad University, Marrakech, Morroco (2011).

[5] A. Barroug, and M.J. Glimcher, J. Orthop. Res. 20, 274 (2002).

[6] R.G.G. Russell, N.B. Watts, F.H. Ebetino, and M.J. Rogers, Osteoporos. Int. 19, 733 (2008).

[7] B.A. Chabner, and J.A. Collins, Cancer and chemotherapy: principles and practice (Philadelphia, Lippincott Co, 1990).

[8] L. Benaziz, A. Barroug, A. Legrouri, A. Lebugle, and C. Rey, J. Colloid Interface Sci. 238, 48 (2001).

[9] A. Barroug, C. Rey, M.J. Fauran, J.C. Trombe, G. Montel, and G. Bonel, Bull. Soc. Chim. Fr. 4, 535 (1985).

[10] C. Rill, Z.I Kolar, G. Kicklbick, H.Th. Wolterbeek, and J.A. Peters, Langmuir 25, 2294 (2009).

[11] E.C. Moreno, M. Kresak, and D.I. Hay, J. Biol. Chem. 257, 2982 (1982).

[12] A. Barroug, A. Legrouri, and C. Rey, Key Eng. Mater. 361-363, 79 (2008). 